\title{
The stuff new species are made of?
}

\author{
Axel Meyer \\ Department of Biology, University of Konstanz, 78457 Konstanz, Germany. e-mail: axel.meyer@uni-konstanz.de
}

\begin{abstract}
A genome-w ide linkage map for the threespine stickleback provides a first glimpse of the evolutionary genetic basis of morphological differentiation in a non-model vertebrate. Within extremely short evolutionary time spans, significant adaptive changes of known ecological consequence seem to have been brought about by a surprisingly small number of loci with major phenotypic effects.
\end{abstract}

Niko Tinbergen, one of the recipients of the 1973 Nobel Prize in Medicine and Physiology, shared it with Konrad Lorenz and Karl von Frisch for their discovery of several fundamental principles of animal behavior, such as the supernormal stimulus and fixed action patterns. Tinbergen also worked on the mating dance of the threespine stickleback (Gasterosteus aculeatus), and like many other researchers after him, he investigated its behavior - and other aspects of its biology - with the expectation that it would teach us generally applicable lessons. Whereas a great deal is already known about many facets of the stickleback's life, it continues to provide important insights into various biological problems, particularly in the fields of ecology and evolutionary biology 1-3.

In a recent issue of Nature, Catherine Peichel and colleagues ${ }^{4}$ introduce the stickleback to a new discipline- evolutionary genomics. Because so much is already known about many aspects of the biology of sticklebacks, they would seem to be a good choice for a study on the genetic nature of morphological evolutionary diversification. The aim of the recent work ${ }^{4}$ was to identify chromosomal regions that contain gene loci (quantitative trail loci (QTL) to be precise) that are responsible for especially interesting phenotypic innovations of documented importance in ecological and evolutionary diversification.

\section{Kinds of sticklebacks}

Sticklebacks are small fishes that live in streams and ponds in the northern temperate zone. One feature that has attracted the attention of biologists is that two interfertile morphs or ecophenotypes-sometimes they are considered to be species - can be recognized within some populations. There are slender, more streamlined 'limnetic' sticklebacks with elongated bodies, relatively large eyes and longer snouts (see figure). They

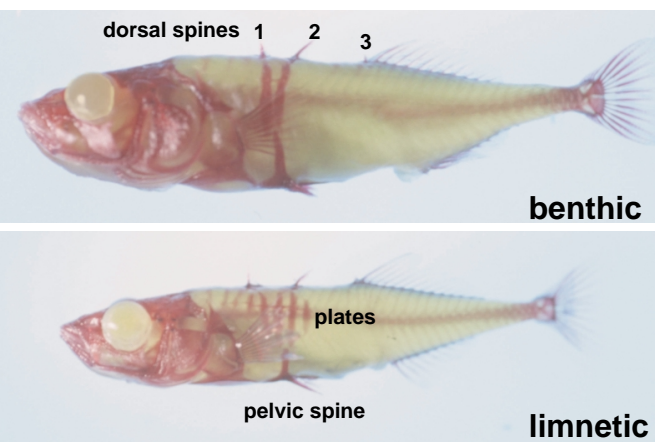

Stickleback morphs. Benthic and limnetic morphs of the species of stickleback studied by Peichel et al. ${ }^{4}$. Bone is stained with alizarin red and cartilage with alcian blue. Note the differences in the proportions between the two types, such as depth of of the number of 'arrow' plates on the side of the body and the relative lengths of the spines in the dorsal and pelvic fins. Figure courtesy of Catherine Peichel and David Kingsley, modified from Nature 414, 901-905 (2001).

live predominantly in open water and have a larger number of gill rakers (protuberances from their branchial skeleton that also holds the gills) that filter food particles, mostly zooplankton, and prevent prey from escaping from the mouth of the fish. Limnetic sticklebacks also have longer spines in the dorsal fin and pelvic fins than benthic morphs and more lateral bony plates covering their flanks. The spines in their fins, and also the bony plates, resembling the armor of a knight, serve to deter potential fish and bird predators.

'Benthic' sticklebacks are deeper bodied, live closer to the shore than their slender cousins, feed on invertebrates, and have a greatly reduced body armor and a decreased number of gill rakers (see figure). Limnetic and benthic morphs in polymorphic fish species are known from several other groups of temperate and tropical freshwater fish, including some salmonids, and also cichlids ${ }^{5}$, an extremely species-rich group of fishes that have repeatedly formed flocks of several hundred endemic species in several lakes in Africa ${ }^{6}$.

\section{Here come the geneticists}

Despite some degree of reproductive isolation between the limnetic and the benthic morphs of sticklebacks in nature ${ }^{3}$, they can bemated easily in the laboratory to produce a viableand fertileFl generation, permitting a genetic analysis to characterize the number and location of genetic loci that contribute to adaptive phenotypic differentiation ${ }^{4}$. A genome-wide linkage map was created by Peichel et al. ${ }^{4}$ based on a cross between a benthic femalestickleback and a limnetic male from Priest Lakein Canada, and a single F1 male was crossed to a second benthic female. Fortunately, of the 281 newly developed microsatellite markers that can be amplified reliably, a high percentage (81\%; 227 markers) were polymorphic in the family of 92 individuals that were included in the study. Sticklebacks have 21 chromosomes and a genomesize of about 0.58-0.70 Gb. While not as compact as the genome of the pufferfish, it is a rather small genome for fishes, and onepolymorphic marker was found for about every 4 centimorgans (cM ), arranged into 26 linkage groups for a total genetic distance of $886 \mathrm{cM}$. More densely placed markers would, of course, be expected to comeup with thesamenumber of linkage groups as chromosomes.

\section{Genes for spines and rakers}

This map permitted the linkage of these markers to evolutionarily relevant traits such as dorsal and pelvic spine lengths, gill raker and lateral plate numbers. The genetic architecture underlying gill raker number and size is somewhat complex. The number of long gill rakers seems to be determined by a large number of loci, each with small effects; however, the number of short gill rakers is controlled by only two major QTL that map to two different linkage groups. The length of the protective spines in the dorsal and the pelvic fins and the number of lateral plates is under the control of a small number of QTL that map to several distinct chromosomes. 
Interestingly, theloci that control thelength of the first and second spinein the dorsal fin map to distinct linkage groups. This suggests that seemingly serially homologous traits (two spines of one fin), whose expression seems to becorrelated within onemorphological structure, can be controlled to a significant extent by two different loci on two different chromosomes. However, the length of the second dorsal spineand that of the pelvic spine maps to adjacent genomic regions, suggesting that these functionally linked traits arealso under the control (possibly pleiotropic) of the same or at least two closely linked loci.

The stickleback linkage map provides tantalizing results and is an important beginning to build on for future work, but does it describe the general genetic nature of evolutionary change? Not enough comparative data are yet available, and as these data are not easy to come by, no generalities have yet emerged. We do have an enticing insight-that both small effects of many genes and large effects of a small number of genes can account for variation in certain characteristics that differentiate species from one another. As Peichel et al. ${ }^{4}$ suggest, some of the findings of the stickleback study might bepartly explained by theexperimental design. By using only one family, one is going to overestimatetheeffect of individual
QTL and underestimate the number of loci that have a significant phenotypic effect. Evolutionary biology is a comparative and historical science. Only through additional studies can generalities, if they indeed exist, be discovered. Where shall we look? Stickle backs might bea good model.

\section{Parallel speciation}

One of the most remarkable aspects of sticklebacks is that the distinctive morphs livesympatrically (in the samelocality) in at least six lakes in British Columbia. The differences between the limnetic and benthic sticklebacks apparently evolved extremely rapidly, independently and repeatedly-in parallel-from marine ancestors that colonized coastal lakes that formed during the last ice age ${ }^{3}$. Parallel speciation shows that evolution repeats itself at the morphological level, but molecular phylogenetic information is necessary to be able to recognize such cases in the first place. Evolutionarily similar morphological solutions to ecological problems, as well as the occupation of equivalent ecological niches in different environments, is receiving attention from evolutionary biologists interested not only in sticklebacks but also in other evolutionary models such as Anolis lizards and cichlid fishes, where some of the most notable instances of parallel evolution in several
African lakes have been documented ${ }^{6-9}$. The exact mechanisms of independent parallel speciation (whether allopatric or sympatric) are debated both in general and in sticklebacks in particular ${ }^{3,10-12}$, but each of these British Columbian lakes offers an independent experiment in parallel innovation in limnetic and benthic morphologies. If thereare genomic rules for diversification at the phenotypic level to be discovered, it is in these remarkable cases of parallel speciation where one might want to look. Are the sameloci used in parallel to bring about the same adaptations, or does evolution recruit completely different genes and/or molecular mechanisms to produce similar phenotypic responses to equivalent ecological challenges?

1. Bakker, T.C.M., Künzler, R. \& Mazzi, D. Nature 401, 234 (1999).

2. Reusch, T.B.H., Häberli, M.A., Aeschlimann, P.B. \& Milinski, M. Nature 414, 300-302 (2001).

3. Rundle, H.D., Nagel, L., Boughman, J.W. \& Schluter, D. Science 287, 306-308 (2000).

4. Peichel, C.L. et al. Nature 414, 901-905 (2001).

5. Meyer, A. Biol. J. Linn. Soc. 39, 279-299 (1990)

6. Stiassny, M.L.J. \& Meyer A. Sci. Am. (February) 64-69 (1999).

7. Wilson, A.B. Noack-Kuhnmann, K. \& Meyer, A. Proc. Roy. Soc. Lond. B 267, 2133-2141 (2000).

8. Kirkpatrick, M. Nature 408, 299-300 (2000).

9. Schluter, D. The Ecology of Adaptive Radiation (Oxford Univ. Press, Oxford, 2000).

10. Johannesson, K. Trends Ecol. Evol. 16, 148-153 (2001).

11. Schluter, D., Boughman, J.W. \& Rundle, H.D. Trends Ecol. Evol. 16, 283-284 (2001)

12. Johannesson, K. Trends Ecol. Evol. 16, 284 (2001). 